\title{
Study of the Deposited Energy Spectra in Silicon by High-Energy Neutron and Mixed Fields
}

\author{
Carlo Cazzaniga $^{\circledR}$, Rubén García Alía ${ }^{\circledR}$, Maria Kastriotou ${ }^{\circledR}$, Matteo Cecchetto ${ }^{\circledR}$, \\ Pablo Fernandez-Martinez ${ }^{\circledR}$, and Christopher D. Frost ${ }^{(}$
}

\begin{abstract}
The energy deposition spectra in a silicon detector have been measured at chip irradiation (ChipIr) and Cern High energy AcceleRator Mixed field (CHARM) facilities. The measurement was possible thanks to a fast electronic chain that can cope with high instantaneous fluxes. A computational study of the energy deposition in a silicon detector allows for the comparison of high-energy spallation facilities dedicated to the irradiation of microelectronics and for the validation of radiation transport models. The measured time structure of the facilities pulses is also presented with an example on how to use this result to correct in the case of large dead times (DTs).
\end{abstract}

Index Terms-Nuclear measurements, particle beams, silicon detector.

\section{INTRODUCTION}

$\mathbf{I}$ RRADIATION tests of microelectronics components to study single event effects (SEE) are performed by industry, research institutes, and academia at dedicated irradiation facilities. In particular, spallation facilities try to reproduce radiation environments of interest, accessing energy ranges in excess of hundreds of $\mathrm{MeV}$. These high energies can be reached only with large-scale accelerators, hence the limited availability worldwide. In Europe, two spallation facilities used for microelectronics testing are chirp irradiation (ChipIr) and Cern High energy AcceleRator Mixed field (CHARM). ChipIr, a beamline at the Rutherford Appleton Laboratory (U.K.), use the spallation of $800-\mathrm{MeV}$ protons of the ISIS accelerator on a tungsten target to produce an atmospheric-like neutron beam [1]. CHARM, a facility at CERN (Switzerland), uses the $24-\mathrm{GeV}$ protons of the PS accelerator on a copper target to produce a mixed field of high-energy hadrons, mainly neutrons, but also protons, pions, and kaons [2].

According to the nature of the radiation fields, ChipIr is mainly dedicated to ground level or flight altitude testing, while CHARM is dedicated to accelerator or space applications. The reason for a detailed cross-calibration of the two

Manuscript received July 4, 2019; revised August 23, 2019 and September 26, 2019; accepted September 26, 2019. Date of publication September 30, 2019; date of current version January 29, 2020.

C. Cazzaniga and C. D. Frost are with ISIS Facility, STFC, Rutherford Appleton Laboratory, Didcot OX11 0QX, U.K. (e-mail: carlo.cazzaniga@ stfc.ac.uk; christopher.frost@stfc.ac.uk).

R. G. Alía, M. Kastriotou, M. Cecchetto, and P. Fernandez-Martinez are with the EN Department, CERN, CH-1211 Genève, Switzerland (e-mail: ruben.garcia.alia@cern.ch; maria.kastriotou@cern.ch; matteo.cecchetto@ cern.ch; pablo.fernandez.martinez@cern.ch).

Color versions of one or more of the figures in this article are available online at http://ieeexplore.ieee.org.

Digital Object Identifier 10.1109/TNS.2019.2944657 is that the limited availability of beam time, the increasing demands for radiation testing of microelectronics, and the multiple applications that an electronic product can have, push for a degree of interchangeability of these kinds of facilities. Experimental and computational methods are needed to compare the results obtained in these different environments.

A first comparison of the two radiation environments was presented in 2018 by Cecchetto et al.[3], using single-event upsets and single-event latch-up measurements on SRAM-based monitors [4]. This article presents a study performed with a silicon detector, and the most interesting advance is the capability to study the deposited energy spectra by high-energy hadrons in silicon. The comparison of Monte Carlo simulations with the measurements allows for a validation of the models of radiation transport and interaction that are used for the characterization of radiation environments and to predict the mechanisms of radiation effects on microelectronics. A second interesting result presented in this article is the measurement of the time structure of the radiation sources that is derived from the different pulsed nature of the accelerators. The time structure of the beam can be important to consider for some applications where the probability of multiple interactions is high.

\section{EXPERIMENTS}

\section{A. Facilities}

ChipIr is a beamline of the second target station (TS2) of the ISIS accelerator. The $800-\mathrm{MeV}$ protons, extracted from the synchrotron, are collided on a tungsten target. The proton beam on TS2, typically running at $40 \mu \mathrm{A}$, is pulsed at $10 \mathrm{~Hz}$, and each pulse is composed by two bunches that are about $70 \mathrm{~ns}$ wide (half maximum) and $360 \mathrm{~ns}$ apart. ChipIr is dedicated and optimized to the testing of microelectronics for SEE. The line of sight looks at a secondary reflector with a $30^{\circ}$ angle with respect to the proton beam, this optics is designed to give some shaping to the neutron spectrum and reduce the contribution of other particles (like charged particles or gammas). The resulting fast neutron spectrum is similar to the atmospheric one up to $800 \mathrm{MeV}$. The flux of neutrons above $10 \mathrm{MeV}$ is $5.6 \cdot 10^{6} \mathrm{~cm}^{-2} \mathrm{~s}^{-1}$ at the front reference position. (This is the position where users can setup their devices under test.) The beam is collimated, and different sizes can be selected by a collimator system. Here, the standard configuration of $70 \times 70 \mathrm{~mm}^{2}$ beam was used. ChipIr is a 
new facility, with the first user campaigns performed in 2017. A first benchmark of the spectrum with activation foils was published in 2018 [5], and the energy deposition spectrum in silicon is studied in this article.

CHARM is a facility at CERN where an extraction line from the PS accelerator delivers $24-\mathrm{GeV}$ protons to a copper target to produce mixed fields of high-energy hadrons (mainly neutrons, pions, and protons). This makes it useful for testing electronics dedicated in particular to the accelerator environment and space. The extraction of a proton pulse (or spill) happens about every $30 \mathrm{~s}$ (the exact time depending on the specific PS super-cycle) and every slow-extracted spill is $350 \mathrm{~ms}$ long. The facility consists of a large irradiation hall with several radial test positions, looking to the target at different angles. A system composed of four shielding blocks (concrete and/or iron) can be remotely moved in between the target and the irradiation positions. By choosing a test position and shielding configuration one can change the radiation field, typically changing the hardness of the spectrum and the relative composition of the mixed field. For this experiment, we used the G0 position without shielding (CuOOOO). G0, having a very wide angle $\left(87^{\circ}\right)$, has one of the softest spectra $(E<1 \mathrm{GeV})$ for kinematics reasons. This position also has a high content of neutrons. In this configuration, the flux of highenergy hadrons with $E>10 \mathrm{MeV}$ was $2.87 \cdot 10^{6} \mathrm{~cm}^{-2} /$ spill.

The radiation environment in CHARM has been extensively characterised using RadMON detectors [2] (measuring HEH fluence, $1-\mathrm{MeV}$ neutron equivalent fluence, and total ionizing dose) and activation measurements [6] (focusing on thermal neutron fluence measurements, as well as the deconvolution of the neutron spectrum in low flux areas of the facility). In this article, the first effort of measuring the mixed-field radiation environment in CHARM through its event-by-event energy deposition distribution, as opposed to the integral quantities mentioned above, is presented.

\section{B. Setup of the Silicon Detector}

The silicon detector is a totally depleted $\mathrm{p}$ - $\mathrm{n}$ junction diode manufactured by Micron Semiconductors Ltd. The geometry of the silicon is square $(2 \mathrm{~mm} \times 2 \mathrm{~mm} \times 140 \mu \mathrm{m})$ and features a ceramic PCB and a metal housing.

The same setup has been used on the two facilities. In the irradiation halls, the detector is connected to a low-noise current amplifier (Cividec C1 [7]) with an analog bandwidth of $2 \mathrm{GHz}$ and a $20-\mathrm{dB}$ gain. From the preamplifier, the coaxial connections are going to the counting rooms. The reverse bias $(+40 \mathrm{~V})$ is given by an ORTEC model 710 bias supply [8]. The signal output is fed to a channel of a CAEN digitizer, 1 GSample/s-10 bits [9]. This is used in oscilloscope mode: all the waveforms triggering above the threshold are recorded, including their timestamps. The waveforms are then analyzed offline so that the deposited energy histograms can be built.

This electronic chain has been designed to provide fast signals for high counting rate applications [10]-[12], where pile-up can be an issue. An example of a measured waveform induced by a single particle interaction in the silicon detector

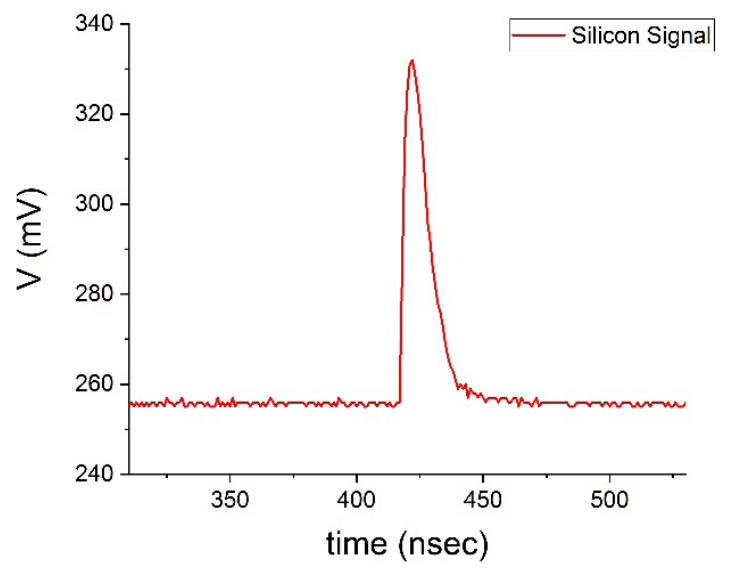

Fig. 1. Example of a single waveform measured by the silicon detector.

is shown in Fig. 1. Here one can see that the signal is $10 \mathrm{~ns}$ wide (FWHM) and returns to the baseline in about $30 \mathrm{~ns}$.

The accelerator start signal is also recorded by a second channel of the same digitizer. This is an NIM logic signal that comes from the extraction of the spill from the synchrotron ring. The time difference between the start and radiation pulses is used to reconstruct the time structure of the source.

\section{ANALYSIS AND RESUltS}

\section{A. Deposited Energy Spectra}

When using a current preamplifier, the collected charge $Q$ is proportional to the area under the waveform $V(\mathrm{t})$, after subtracting the baseline

$$
Q=\frac{1}{G} \times \int \frac{V(t)}{R} d t
$$

where $G$ is $20 \mathrm{~dB}$, as calibrated from the manufacturer, and $R$ is $50 \Omega$.

The deposited energy $E_{\text {dep }}$ is given by the number of electron-hole pairs produced $(Q / e)$, where $e$ is the elementary charge, and the energy necessary for pair production in silicon $\left(E_{\mathrm{eh}}=3.76 \mathrm{eV}\right)$

$$
E_{\mathrm{dep}}=\frac{Q}{e} E_{\mathrm{eh}} .
$$

The measured deposited energy spectra are presented in Fig. 2 for both CHARM (G0) and ChipIr. The energy threshold used in the measurements is $2 \mathrm{MeV}$ for CHARM and $8 \mathrm{MeV}$ for ChipIr. The reason for this difference is that on ChipIr, the accelerator spills are much shorter (see Section III-B). Therefore, the probability of pile up is higher, and this hinders the capability to distinguish low deposited energy signals, where the detection probability is higher as well as the contribution of $\gamma$-ray-induced signals.

For ChipIr, the counts are given per second, as the accelerator has a constant extraction frequency of $10 \mathrm{~Hz}$ on TS2. For CHARM, counts are given per spill, as the frequency is not necessarily constant. Also, since the copper target at CHARM is taken out of the beam for some periods, we considered only the production spills, when the beam was on target. 

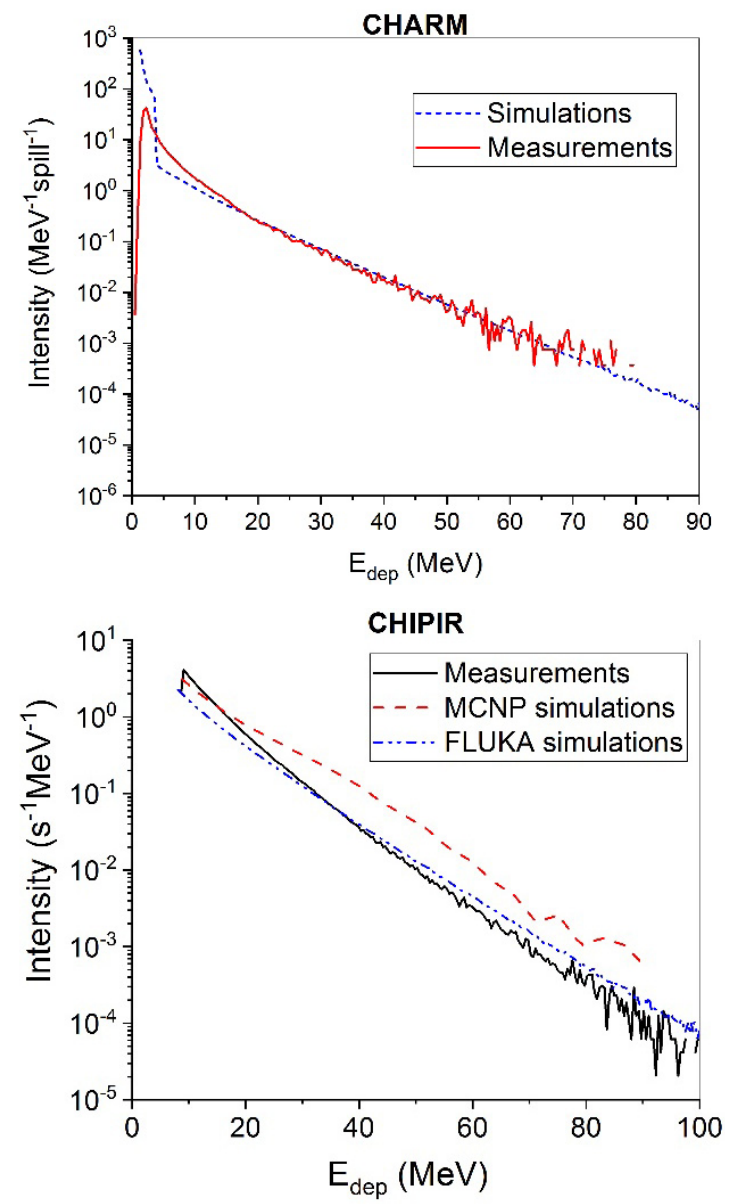

Fig. 2. Deposited energy spectra measured with a silicon detector at CHARM (top) and ChipIr (bottom). A comparison with Monte Carlo simulations of radiation transport is presented.

The measurements are compared to Monte Carlo simulations and results are shown in the same Fig. 2.

The Monte Carlo model of the neutron and mixed-field transport and interaction with silicon has been developed with FLUKA [13]. This takes as input source the spectra provided by the facilities (see Fig. 3).

The input spectra of Fig. 3 can be also found in [5] and [14]. They are the spectra of high-energy hadrons that are neutrons for ChipIr and a mixed field for CHARM. Both spectra are produced by spallation mechanism; therefore, they are both white and extending to hundreds of MeV. Different shapes are caused by many factors: 1) the target material; 2) the accelerator energy; 3 ) the angle of the line of sight with respect to the primary beam; and 4) the radiation transport in materials close to the target and in the line of sight.

Examining the deposited energy spectrum, for neutrons, the contribution to the spectrum is due to several possible nuclear interaction mechanisms with silicon nuclei. Nuclear cross sections from the TENDL library are shown in Fig. 4 [15]. In the low-energy part (few $\mathrm{MeV}$ ), the total cross section is dominated by elastic (kinetic energy is conserved) and inelastic scattering (the nucleus is left on an excited state and $\gamma$-rays are emitted). Above $4 \mathrm{MeV},(n, \alpha)$ and $(n, p)$ threshold reactions

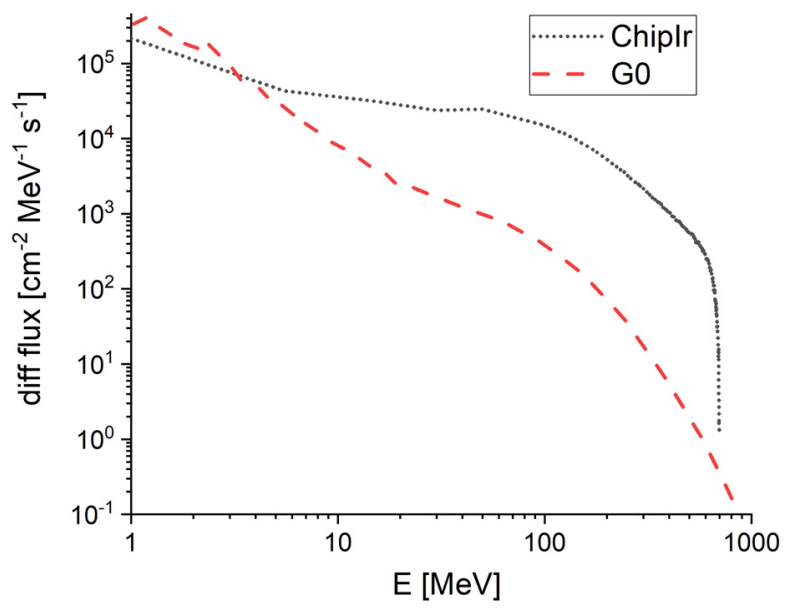

Fig. 3. Comparison of ChipIr and CHARM (G0) high-energy hadrons spectra as found in [5] and [14]. Note that spectra are normalized to time (per second); ChipIr spectrum is more intense because the repetition rate of the spill is faster.

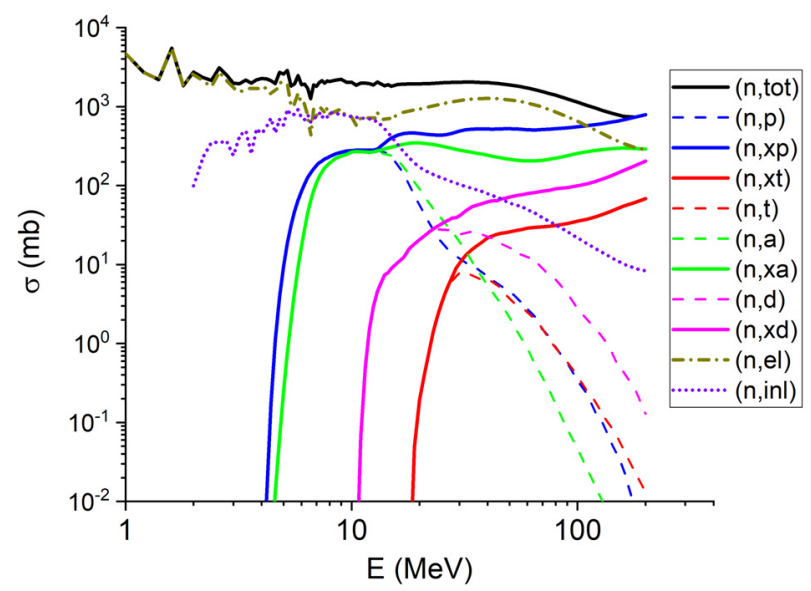

Fig. 4. Neutron nuclear cross sections of Si-28 for the production of recoil and secondary radiation from the TENDL library [15].

are open; above $10 \mathrm{MeV}$ more reactions are open, like $(n, d)$ and $(n, t)$; more complex reactions, like $(n, \mathrm{xp}),(n, \mathrm{xa})$, where more than two products are emitted, dominate at high energies.

For protons and pions, the most probable mechanism of energy deposition is the direct ionization that contributes to the lowest part of the spectrum (few $\mathrm{MeV}$ ). At high-energy nuclear reactions similar to the ones induced by neutrons are possible.

Fig. 5 shows the simulation of the contribution of neutrons protons and pions of the CHARM mixed field to the total deposited energy spectrum. It is interesting to see that protons dominate at low energy $\left(E_{\mathrm{dep}}<5 \mathrm{MeV}\right)$, neutrons at intermediate energy $\left(E_{\text {dep }}<40 \mathrm{MeV}\right)$, and pions at high energy $\left(E_{\mathrm{dep}}>40 \mathrm{MeV}\right)$.

Commenting again the comparison of Fig. 2, one can see a very good agreement of simulations and measurements for most part of the spectrum. In particular the agreement for $E_{\text {dep }}>20 \mathrm{MeV}$ is excellent. For both facilities, the measurements exceed the simulation in the range $5-15 \mathrm{MeV}$. This can be due to two reasons: 1) only neutrons above $20 \mathrm{MeV}$ 


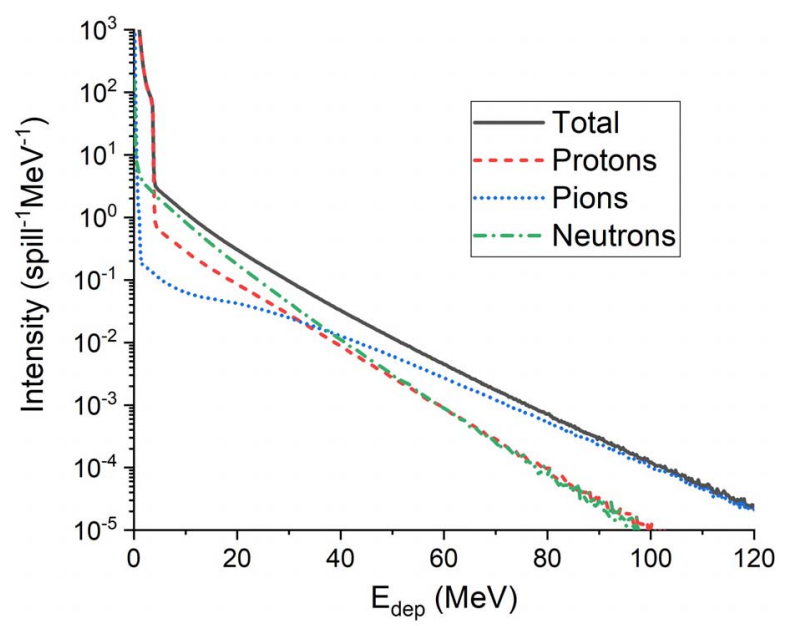

Fig. 5. FLUKA simulation of the deposited energy spectrum at CHARM separated for the contribution of neutrons, protons, and pions.

generate event-by-event energy deposition in FLUKA due to the group treatment below $20 \mathrm{MeV}$ and 2) the input spectra are impinging only on the detectors, and surrounding structures can be responsible for an increased scattering.

For ChipIr, a Monte Carlo model has been built also using MCNP [16] [Fig. 2, (bottom)]. One can notice that, despite the result being relatively close, the agreement with the measurements is not as good as for the FLUKA model. As we expected, for hadronic models (neutrons above $20 \mathrm{MeV}$ ) FLUKA is more accurate than MCNP. In fact, FLUKA was developed with particular aim to high-energy physics experiments and applications $(E>20 \mathrm{MeV})$, while MCNP was originally dedicated to the application of nuclear physics (e.g., fission reactors, $E<20 \mathrm{MeV}$ ).

The highly satisfactory agreement between the simulated and measured energy deposition distributions in such spallation fields is essential, as modeling tools are often used to simulate the SEE rate in complex environments such as that present in high-energy accelerators. Indeed, recent studies have focused on describing the impact of a variety of radiation fields not only in terms of their particle energy (or LET) spectra as done in standard approaches but rather through their deposited energy in sensitive volumes representative of those involved in SEE induction. Applications of such studies include the possible use of proton data as a proxy for heavy ion sensitivity [17], [18], the impact of high- $Z$ materials on the energy dependence of proton and neutron SEE cross sections and the respective RHA implications [14], and the contribution of heavy ion nuclear interactions to the sub-LET threshold cross section region [19]. Though successfully benchmarked in terms of SEE cross section for the beams and mixed fields available experimentally, a satisfactory event-by-event energy deposition distribution agreement between experiment and simulation, as that shown in Fig. 2 for the FLUKA Monte Carlo code provides a much more direct validation of such models at an energy deposition level and therefore independent of the assumed SEE criterion.
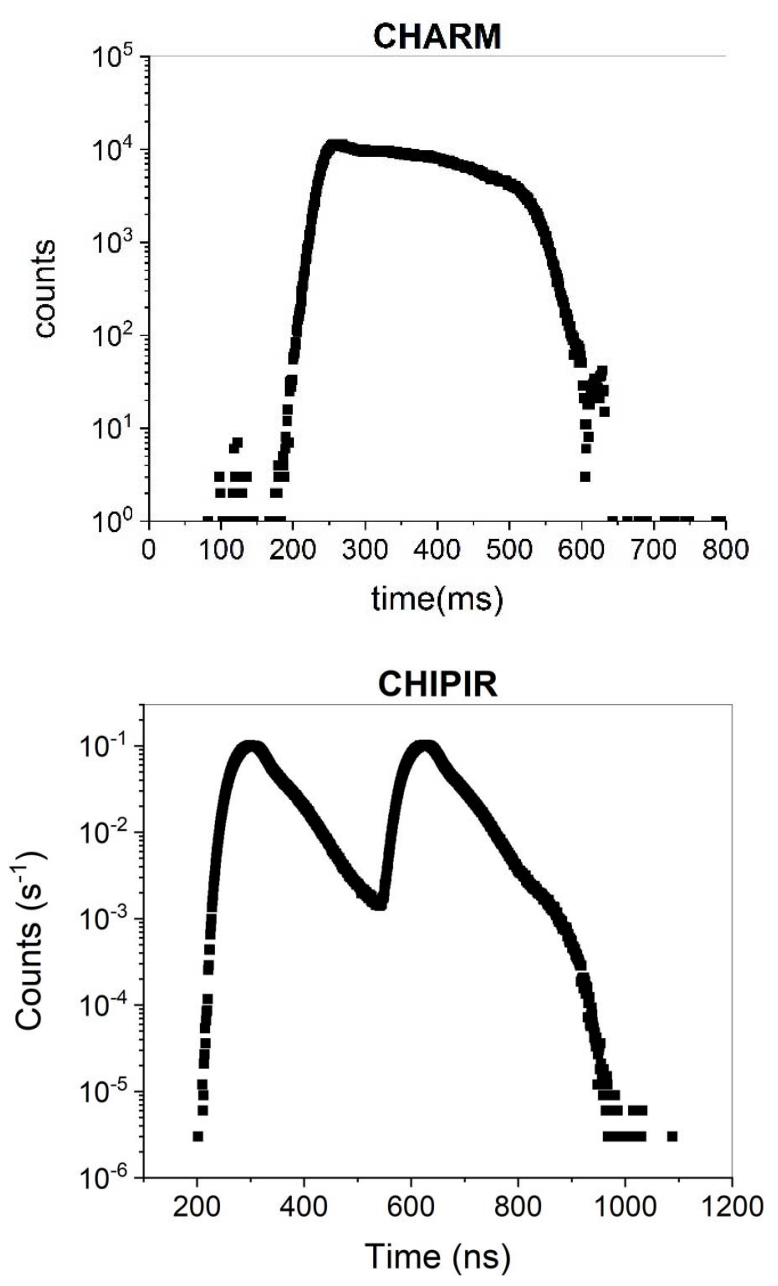

Fig. 6. Time structure of the pulsed beams at CHARM (top) and ChipIr (bottom) measured by the silicon detector. Note here the fact that different time units are used in each case.

In addition, results in Fig. 5 show that in the mixed-field environment and for the considered charge collection size, protons and neutrons have similar energy deposition distribution curve shapes, whereas pions, though being less abundant, in fact, dominate the energy deposition above roughly $40 \mathrm{MeV}$. The satisfactory agreement between the experimental and simulated energy deposition curves also in this high-deposited energy regime provides confidence in applying Monte Carlo tools to SEE mixed-field calculations, grasping the impact of the variety of particles and energy ranges involved in the energy deposition.

\section{B. Source Time Structure Analysis}

The time structure of the irradiation field is measured by building histograms of $\Delta t$, the difference of the signal timestamp and associated start signal timestamp from the accelerator extraction. The result is presented in Fig. 6 for both CHARM and ChipIr, with different time units in the $x$-axis.

The digital electronic chain measures with great accuracy the timing of signals. In fact, the accuracy on the timestamp of this particular digitizer is $8 \mathrm{~ns}$ that is more than enough for the purpose of this analysis. 

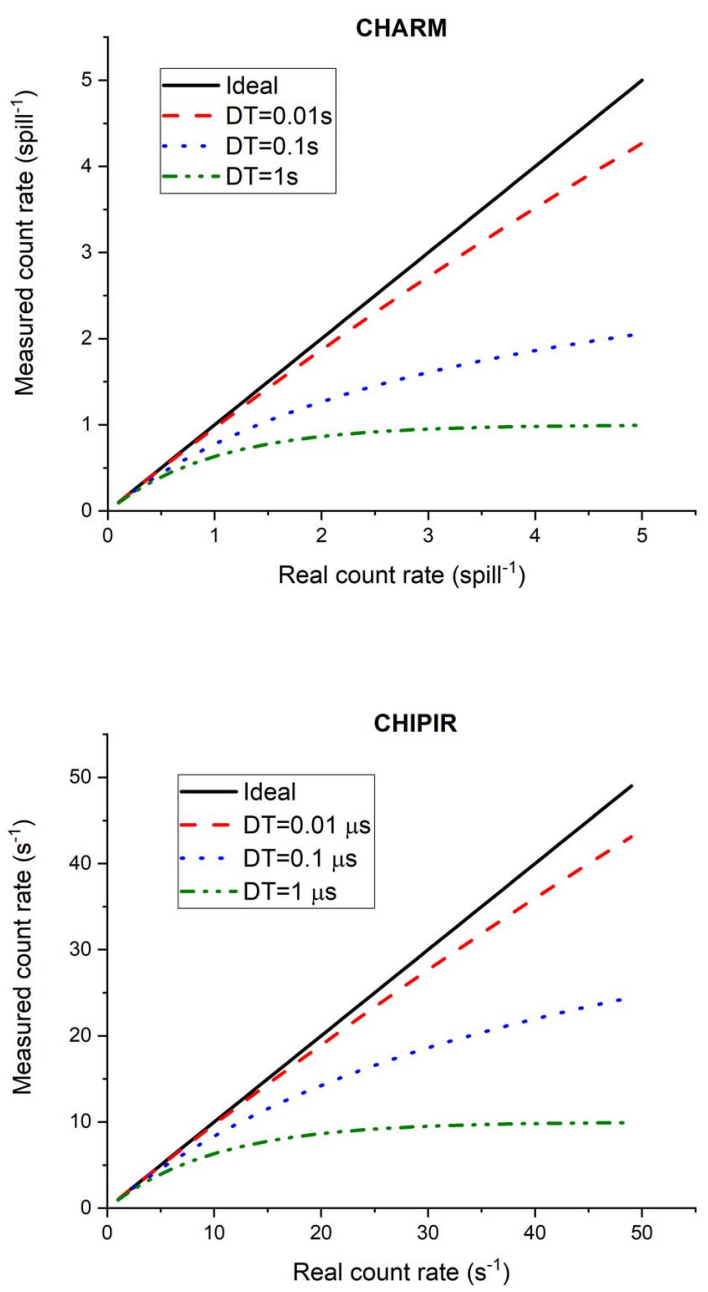

Fig. 7. Example case study of the calculation of real versus measured count rates using the time structure results presented in Fig. 6 for CHARM (top) and ChipIr (bottom), for different DTs.

One can notice that on CHARM the spill is much more stretched in time, as it spans on about $400 \mathrm{~ms}$ with a quasiuniform intensity. It is rather flat even if after reaching the maximum (about $200 \mathrm{~ms}$ after the start signal) a gradient can be noticed.

On ChipIr, the double bunch structure is clear. Each proton bunch is $70 \mathrm{~ns}$ wide, and their time structure would be symmetrical on the target. The strong asymmetry is due to the time of flight necessary for the neutrons to travel the 15-m flight path so that clearly lower energy neutrons take more time for travel. This, of course, cannot be seen on the CHARM pulse.

The time structure results can be used to correct experimental results when dead time (DT) or pile-up is important. In Fig. 7, we present a case study for each facility. Here, we calculate the measured count rate as a function of the expected real count rate. If the flux were constant, it would be easy to find this with an analytical solution. Taking this complex time structures as input, we need to use a Monte Carlo approach to compute the solution. The algorithm extracts events with random time-tags using the input time distributions. It then rejects the events that fall inside the DT of another event (that is considering a nonparalyzable system). For the case study, we had to choose an event rate and DT values such that we are in the most interesting conditions. In fact, we need to highlight that the facilities are designed to have fluxes such that these corrections are not needed for most of the cases in SEE testing. If, as in most SEE testing cases, the cross section and the DT are low enough, the measured and real count rate will be the same, as the ideal linear behavior in Fig. 7. If, on the other extreme, the DT is too large with respect to the size of the accelerator pulse width, we reach a trivial situation where the measured count rate saturates to one count per spill. The interesting cases, as chosen in the analysis presented in Fig. 7, are when the DT is a reasonable fraction of the pulse width, and when the interaction cross section is such to give several events per spill. In these cases, we see a behavior that is linear at lower counting rates and then it curves to underestimate the real count rate. Some applications, where the interaction cross section is high, as, for example, the testing of macroscopic power transistors, or the testing of radiation detectors, might indeed need to use this kind of correction.

\section{CONCLUSION}

A silicon detector has been used at CHARM and ChipIr to study the deposited energy distribution in silicon of high-energy neutrons and mixed fields. The fast electronic chain that has been used, preamplifiers and digital acquisition, is able to cope with high fluxes. The deposited energy spectra have been collected and compared to simulations. The agreement with the FLUKA model looks very promising, and it will be further examined, aiming at further improvements, using new software releases due in the coming years, while it has been demonstrated that an MCNP model is less performing. Therefore, this method can be used to validate Monte Carlo models that can then be used to simulate mechanisms of single event effects (SEE). We highlight that both for ChipIr and CHARM, the presented results are the first experimental study of the deposited energy spectrum in silicon, which of course is particularly relevant for its application in microelectronics. Using this setup, we could measure the deposited energy event-by-event; this is a clear addition to other measurements (e.g., RADMON, SEE monitors) where one can only count the events above the threshold.

The detector also successfully measured the time structure of the pulsed fields that can be important information for particular applications that suffer pile-up or have a large DT.

\section{REFERENCES}

[1] C. Cazzaniga and C. D. Frost, "Progress of the scientific commissioning of a fast neutron beamline for chip irradiation," J. Phys., Conf. Ser, vol. 1021, no. 1, May 2018, Art. no. 012037.

[2] J. Mekki, M. Brugger, R. G. Alia, A. Thornton, N. C. Dos S. Mota and S. Danzeca, "CHARM: A mixed field facility at CERN for radiation tests in ground, atmospheric, space and accelerator representative environments," IEEE Trans. Nucl. Sci., vol. 63, no. 4, pp. 2106-2114, Aug. 2016.

[3] M. Cecchetto et al., "SEE flux and spectral hardness calibration of neutron spallation and mixed-field facilities," IEEE Trans. Nucl. Sci., vol. 66, no. 7, pp. 1532-1540, Jul. 2019.

[4] R. Harboe-Sorensen et al., "From the reference SEU monitor to the technology demonstration module on-board PROBA-II," IEEE Trans. Nucl. Sci., vol. 55, no. 6, pp. 3082-3087, Dec. 2008.

[5] D. Chiesa et al., "Measurement of the neutron flux at spallation sources using multi-foil activation," Nucl. Instrum. Methods Phys. Res. A, Accel. Spectrom. Detect. Assoc. Equip., vol. 902, pp. 14-24, Sep. 2018. 
[6] T. Oyama et al., "Measurement and calculation of thermal neutrons induced by the $24 \mathrm{GeV} / \mathrm{c}$ proton bombardment of a thick copper target," Nucl. Instrum. Methods Phys. Res. B, Beam Interact. Mater. At., vol. 434, pp. 29-36, Nov. 2018.

[7] CIVIDEC Catalogue. Accessed: Jul. 4, 2019. [Online]. Available: https://cividec.at/

[8] ORTEC Catalogue. Accessed: Jul. 4, 2019. [Online]. Available: https://www.ortec-online.com/

[9] CAEN Catalogue. Accessed: Jul. 4, 2019. [Online]. Available: https://www.caen.it/

[10] M. Rebai et al., "Time-stability of a Single-crystal Diamond detector for fast neutron beam diagnostic under alpha and neutron irradiation," Diamond Rel. Mater., vol. 61, pp. 1-6, Jan. 2016.

[11] C. Cazzaniga et al., "Single crystal diamond detector measurements of deuterium-deuterium and deuterium-tritium neutrons in Joint European Torus fusion plasmas," Rev. Sci. Instrum., vol. 85, no. 4, Apr. 2014, Art. no. 043506.

[12] C. Cazzaniga et al., "Characterization of the high-energy neutron beam of the PRISMA beamline using a diamond detector," J. Instrum., vol. 11, Jul. 2016, Art. no. 07012
[13] G. Battistoni et al., "The FLUKA code: Description and benchmarking," in Proc. AIP Conf., Batavia, IL, USA, vol. 896, 2007, pp. 31-49.

[14] R. G. Alía et al., "SEL hardness assurance in a mixed radiation field," IEEE Trans. Nucl. Sci., vol. 62, no. 6, pp. 2555-2562, Dec. 2015.

[15] A. J. Koning and D. Rochman, "Modern nuclear data evaluation with the TALYS code system," Nucl. Data Sheets, vol. 113, no. 12, pp. 2841-2934, Dec. 2012.

[16] C. J. Werner, ed. (2017). MCNP Users Manual-Code Version 6.2 LA-UR-17-29981. [Online]. Available: https://laws.lanl.gov/vhosts/ mcnp.lanl.gov/pdf_files/la-ur-17-29981.pdf

[17] R. Ladbury, J.-M. Lauenstein, and K. P. Hayes, "Use of proton SEE data as a proxy for bounding heavy-ion SEE susceptibility," IEEE Trans. Nucl. Sci., vol. 62, no. 6, pp. 2505-2510, Dec. 2015.

[18] R. G. Alía et al., "Simplified SEE sensitivity screening for COTS components in space," IEEE Trans. Nucl. Sci., vol. 64, no. 2, pp. 882-890, Feb. 2017.

[19] R. G. Alía et al., "Proton dominance of sub-let threshold GCR see rate," IEEE Trans. Nucl. Sci., vol. 64, no. 1, pp. 388-397, Jan. 2017. 